\title{
Influence of Near-Surface Blisters on Deuterium Transport in Tungsten
}

\author{
J. Bauer, ${ }^{\mathrm{a}, \mathrm{b}}$, T. Schwarz-Selinger ${ }^{\mathrm{a}}$, K. Schmid ${ }^{\mathrm{a}}$, M. Balden ${ }^{\mathrm{a}}$, A. Manhard ${ }^{\mathrm{a}}$, \\ U. von Toussaint ${ }^{\mathrm{a}}$ \\ ${ }^{a}$ Max-Planck-Institut für Plasmaphysik, 85748 Garching, Germany \\ ${ }^{b}$ Technische Universität München, 85748 Garching, Germany \\ johannes.bauer@ipp.mpg.de
}

\begin{abstract}
The effect of near-surface blisters on deuterium transport in tungsten is studied by means of nuclear reaction analysis (NRA) and scanning electron microscopy (SEM). Gentle deuterium plasma loading of different durations and subsequent NRA depth profiling is performed in heavily pre-blistered and unblistered areas on self-damaged tungsten samples. Comparison of the deuterium depth profiles reveals a considerable reduction of the deuterium transport into the bulk due to the presence of near-surface blisters. SEM and NRA results identify the enhanced re-emission of deuterium from the sample due to open blisters as the underlying mechanism which reduces the deuterium flux into the bulk. Based on a simple analytical hydrogen retention model, the re-emitted deuterium flux by open blisters in the here conducted experiment, is determined to be $80 \%$ of the implanted deuterium flux. In addition, the deuterium flux into the bulk is reduced by $60 \%$ compared to the unblistered case. As a consequence, deuterium retention studies carried out under blister-facilitating conditions should account for surface morphology which reduce the deuterium uptake.
\end{abstract}

\section{INTRODUCTION}

Due to its favorable material properties tungsten is the designated candidate for plasma facing components in future fusion devices. During plasma operation high ion and neutral fluxes of hydrogen isotopes with energies up to $\mathrm{keV}$ are expected to impinge on the first wall [1] in addition to energetic neutron radiation [2] and power loads of up to $20 \mathrm{MW} / \mathrm{m}^{2}$ [3]. The naturally low hydrogen retention in tungsten can be dramatically increased by the creation of defects in the bulk by dense displacement cascades caused by fast neutrons and close to the surface by energetic ion irradiation. In experiments with linear plasma devices, which try to simulate the divertor conditions, the formation of blisters accompanied with an increase in hydrogen retention has been observed [4, 5]. Since the retention of tritium needs to be kept as low as possible in order to provide efficient fueling and to stay below the safety limit, the appearance of blisters is assumed to be unfavorable. Therefore, blistering of tungsten by hydrogen isotopes has been studied extensively, mainly with the intention to understand the formation mechanism of blisters and to develop strategies to avoid them. The appearance of blisters is influenced by many different parameters, e.g. sample temperature [6, 7], ion energy [8], ion flux [9, 10], ion fluence [8], grain orientation [11], material grade [12] and surface finish [13]. As large as the parameter space which determines blistering, as large is the variety of shapes in which blisters occur. With sizes and depths ranging from a few $\mathrm{nm}$ up to tens of $\mu \mathrm{m}$, from the location at grain boundaries to intra grain blisters [14, 15], from circular, cone shaped to flat table-like shaped [16]. Accompanied with the observation of the different kinds of blisters several models such as vacancy-clustering [17], dislocation loop punching [17] or gliding in low-indexed slip systems [16] have been proposed to explain the growth of the particular blisters. Despite this large endeavor to understand blistering, only sparse attempts have been made to study the influence of blisters on deuterium transport explicitly. In some 
studies a reduced or saturating deuterium retention in tungsten was measured by observing blister and crack formation at the same time [18, 19, 20]. From those results it was speculated that ruptured blisters and cracks can lead to an increased re-emission of deuterium from the samples. Qualitatively, the effect of near-surface blisters on deuterium transport was studied by Gao et al. [21]. From the observed suppression of blister formation in larger depth due to the existence of blisters in shallow depth a reduction of the deuterium flux into the bulk was indirectly inferred.

Several possible direct and indirect processes in which blisters can affect hydrogen transport in tungsten are depicted in figure 1. The most apparent one is a direct encounter of a diffusing hydrogen atom with a blister. In case of a closed blister, shown in figure 11(a), the hydrogen atom transits from the bulk to the blister surface, recombines and desorbs as molecule into the blister cavity. At low temperature and insufficient hydrogen pressure inside the blister the reverse process is suppressed. Hence a closed blister represents a trap with a high capacity to capture hydrogen. The contrary is the case, when the blister possesses a connection to the surface as shown in figure 1 (b). A diffusing hydrogen atom which hits an open blister, also transits to the inner blister surface, recombines and desorbs, but instead of being trapped it can leave the sample. Thus, ruptured blisters enhance the re-emission of hydrogen from the sample. In addition, the presence of blisters increase the probability for the hydrogen atom to be re-emitted at the sample surface, shown in figure1 1(c), relative to the probability to diffuse into the bulk, shown in figure 1 (e). Let's assume a hydrogen atom is implanted into a blister cap where it performs a random walk. In order to pass the blistered zone and enter the bulk, the atom needs to diffuse along the blister cap to the rim of the blister where the cap connects to the bulk. Out of geometrical reasons, e.g., the ratio of blister size to cap thickness, this process seems less likely than the encounter of the sample surface or the inner blister surface. A by-product of blister formation is an increased dislocation density in the proximity of the blister [22] as indicated in figure 1 (d). Hence the probability of a diffusing hydrogen to be captured by a dislocation is increased in a blistered sample compared to an unblistered one. Some of the described processes enhance locally the retention, as found in literature. However, all the described processes reduce the hydrogen flux into the bulk, either by enhanced trapping or by enhanced re-emission of hydrogen and should therefore alter the hydrogen uptake in particular in larger depth.

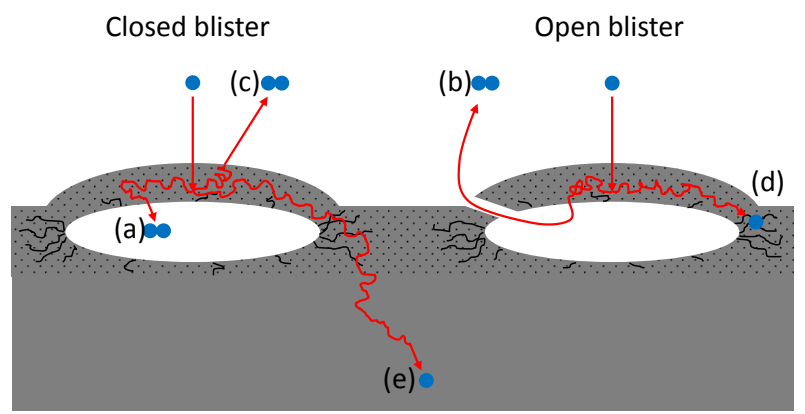

Figure 1: Different processes of blisters influencing the hydrogen transport in tungsten (a) trapped in a closed blister, (b) released through an open blister, (c) enhanced re-emission at the surface, (d) trapped by dislocations, (e) diffusion into the bulk.

In this article, the influence of near-surface blisters on the deuterium transport and retention in tungsten is studied directly and quantitatively in a well-defined laboratory experiment. In order to exclude the effect of an evolving near-surface blister structure on the deuterium transport and the retention, the blisters are created before the deuterium is injected into the 
tungsten samples. The surface morphology of the samples is investigated with scanning electron microscopy (SEM) to obtain information on the blister size, coverage and area density. The depth of the blisters is determined on a cross section prepared by FIB milling. The deuterium is inserted into the samples by gentle plasma loading to avoid the formation of additional defects or blisters. An artificial defect-rich layer, created before plasma exposure, serves as a getter layer which captures deuterium atoms diffusing beyond the depth of the near-surface blisters. The deuterium transport respectively the propagation of the diffusion front in the defect-rich layer and the total deuterium retention is monitored by nuclear reaction analysis (NRA). In order to highlight the influence of near-surface blisters, the deuterium transport and retention in a blistered and unblistered area on the very same sample are compared.

\section{EXPERIMENT}

Hot-rolled tungsten samples of $15 \times 12 \mathrm{~mm}^{2}$ with a thickness of $0.8 \mathrm{~mm}$ and a specified purity of $99.97 \mathrm{wt} . \%$ manufactured by Plansee SE are used for the experiments. The samples are recrystallized at $2000 \mathrm{~K}$ for $5 \mathrm{~min}$ in ultra-high vacuum to anneal inherent defects and increase the grain size of the material. Subsequently the samples are grinded by abrasive paper of decreasing grain size followed by electrochemical polishing in $1.5 \mathrm{wt} . \% \mathrm{NaOH}$ to a mirror-like finish.

In the first step an area of near-surface blisters is created on the specimen by hydrogen ion implantation at low temperature. The implantation is carried out with the Duoplasmatron ion source in the Dual Beam Experiment (DBE) [23], which provides a mass and energy separated beam of deuterium or protium ions. In addition, the experiment has been upgraded with a liquid nitrogen sample cooling system. Deuterium and protium ions with an energy of $3.0 \mathrm{keV} /(\mathrm{D}, \mathrm{H})$ and a flux of $1 \times 10^{18}(\mathrm{D}, \mathrm{H}) / \mathrm{m}^{2} \mathrm{~s}$ are consecutively implanted under normal incidence into the tungsten samples at a temperature of $150 \mathrm{~K}$ up to a fluence of $1 \times 10^{22}(\mathrm{D}, \mathrm{H}) / \mathrm{m}^{2}$ each. Defocusing the ion beam results in a truncated beam profile and a uniform implantation spot with a diameter of approximately $6.5 \mathrm{~mm}$ in which the blisters are created. The sequential implantation of different hydrogen isotopes is the result of a hydrogen isotope exchange study conducted earlier on the samples, which is not subject of the current article.

In the second step, after warm-up to room temperature, the samples are heated ex-situ in the TESS setup [24] to $923 \mathrm{~K}$ in vacuum with a heating rate of approximately $15 \mathrm{~K} / \mathrm{s}$. At this specific temperature no release of retained protium and deuterium is observed anymore, as has been verified by thermal desorption spectroscopy (TDS) during heat-up. In addition, the temperature is low enough to preserve the near-surface blister structure.

In the third step almost the complete sample surface, except for a $0.5 \mathrm{~mm}$ broad frame at the sample edge, is irradiated by $20 \mathrm{MeV}$ tungsten ions. The irradiation is conducted at room temperature up to fluence of $7.9 \times 10^{17} \mathrm{~W} / \mathrm{m}^{2}$ in the TOF beamline of the $3 \mathrm{MV}$ tandetron tandem accelerator as described by Schwarz-Selinger [25]. The tungsten self-implantation creates a defect-rich layer of approximately $2.0 \mu \mathrm{m}$ depth with a maximum at $1.4 \mu \mathrm{m}$ according to SRIM 2013 simulations (Detailed Calculation with full Damage Cascades). Assuming a displacement energy of $90 \mathrm{eV}$ for tungsten [26] a displacement damage level of $0.5 \mathrm{dpa}$ is achieved in the broad peak, which exceeds the reported threshold of $0.4 \mathrm{dpa}$ [27] at which saturation of radiation induced defects occurs. Hence a $2.0 \mu \mathrm{m}$ defect-rich damaged layer with a approximately constant trap concentration is created which serves as a getter layer for the deuterium introduced to the samples in the next step.

Finally, the samples are exposed to a deuterium plasma in the PlaQ setup [28] for 2, 5, 20, 40 and $72 \mathrm{~h}$. During the exposure the samples are kept at $450 \mathrm{~K}$ and on floating potential, resulting in an ion energy of approximately $15 \mathrm{eV}$ and a flux of $6.3 \times 10^{19} \mathrm{D} / \mathrm{m}^{2} \mathrm{~s}$. The majority of the flux is carried by $\mathrm{D}_{3}^{+}(94 \%)$ and a minority by $\mathrm{D}_{2}^{+}(3 \%)$ and $\mathrm{D}^{+}(3 \%)$. Thus, the majority of deuterium atoms penetrating the surface possesses an energy of approximately $5 \mathrm{eV} / \mathrm{D}$. The 
low ion energy and the elevated temperature are chosen to avoid the creation of additional blisters during plasma loading. Furthermore, thermal degassing at room temperature during storage can be neglected if the plasma exposure is conducted at $450 \mathrm{~K}$.

After the exposure of the samples to different deuterium fluences, which results in the decoration of the traps in the previously created damaged layer, depth profiling with nuclear reaction analysis is conducted. The method exploits the $\mathrm{D}\left({ }^{3} \mathrm{He}, \mathrm{p}\right)^{4} \mathrm{He}$ reaction [29, 30] to gain information on the deuterium retained in the blistered and the unblistered regions. The depth profiling is performed at the $3 \mathrm{MV}$ tandetron tandem accelerator at IPP Garching in the RKS setup using ${ }^{3} \mathrm{He}$ ions of eight different energies ranging from $500 \mathrm{keV}$ up to $4500 \mathrm{keV}$ [31]. The ${ }^{4} \mathrm{He}$ and the protons released in the nuclear reaction are measured under a scattering angles of $102^{\circ}$ and $135^{\circ}$, respectively. The acquired spectra are post-processed by SIMNRA 6.8 [32] and NRADC [33] to obtain the deuterium depth profiles and the amount of retained deuterium within the first $7.2 \mu \mathrm{m}$.

Between the different steps described above, SEM images are recorded to monitor changes in the surface morphology of the samples. Before the samples are exposed to the deuterium plasma a FIB cut through several grains is performed to determine the depth range at which the blisters occur.

\section{Results AND Discussion}

\section{A. Surface Morphology}

\section{A.1 Sample Preparation}

In between the different sample preparation steps, SEM is conducted at the identical location to monitor the surface morphology and to detect changes in the near-surface blister structure. Figure 2 (a) shows the backscattered electron image of the surface after keV hydrogen ion implantation at low temperature and subsequent warm-up to room temperature. The individual blisters formed during the implantation can hardly be recognized and individual grains are difficult to distinguish due to the low grain contrast in the image. Nevertheless, the grain contrast itself yields additional information on the sample. When the primary electron beam impinges on a grain which is oriented in a way that a set of crystal planes is aligned parallel to the incident beam, the electrons are channeled deeper into the bulk. As a consequence the backscattering event occurs in greater depth and the probability of the scattered electron to leave the sample is therefore reduced. If the crystal planes of the grain and the primary electron beam are not in parallel, electron channeling is suppressed and the penetration depth of the electrons is lower. Hence backscattering takes place closer to the surface and the probability of the scattered electron to leave the sample is higher, which results in an increased brightness of the backscattered electron image. A low grain contrast in the backscattered electron image is an indication for a distortion of the near-surface crystal structure which reduces electron channeling. Severe damage of the sample surface is anticipated for several reasons. First of all, the implantation energy of the hydrogen ions is sufficient to displace tungsten atoms from their original lattice positions and create vacancy-interstitial pairs [34]. Secondly, the blisters possess regions with compressive and tensile strain which deforms the tungsten lattice [7]. Thirdly, dislocations in the proximity of the blisters are created [22] and finally, the large hydrogen concentration in the implantation zone itself probably also deforms the tungsten lattice.

After annealing the samples at $923 \mathrm{~K}$, shown in figure 2(b), the grain contrast improves significantly and individual blister can be observed much clearer compared to image (a). The blisters appear within the grains and possess a predominately circular shape. Furthermore, the size of the blisters as well as the areal density differs from grain to grain suggesting a dependence on the grain orientation with respect to the surface. This becomes even more apparent in figure 2 (d) which shows a larger area of the blistered region. The increase of the 


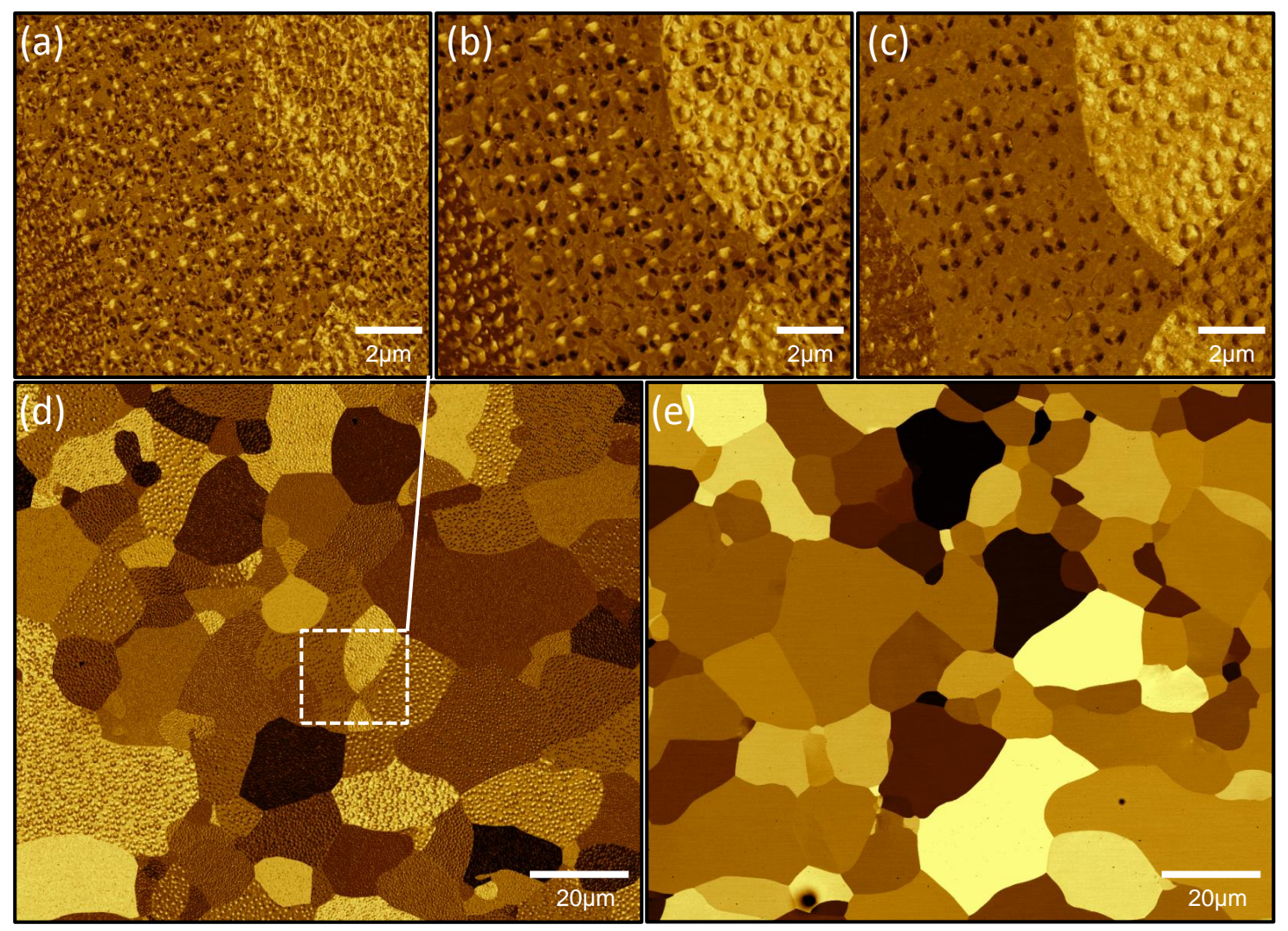

Figure 2: SEM images recorded during sample preparation, sensitive to backscattered electrons. (a) after D and $\mathrm{H}$ implantation at $150 \mathrm{~K}$, (b) after annealing to $923 \mathrm{~K}$, (c) after self-damaging with $20 \mathrm{MeV}$ tungsten ions. (d) and (e) comparison between the blistered and unblistered area before deuterium plasma exposure. 
grain contrast observed after the annealing step indicates the restoring of the crystal structure close to the surface.

The SEM image in figure 2(c), recorded after irradiation of the sample with tungsten ions of an energy of $20 \mathrm{MeV}$, shows a slightly reduced grain contrast compared to image (b) and less prominent blisters. The identification of individual blisters, especially on the grain at the left hand side in the image (c), becomes harder. It also appears that some of the blisters have vanished or shrunk below the detection limit of the SEM.

Despite the annealing and the self-damaging of the blistered samples, the blister structure is mainly preserved. The blister coverage and the areal density of blisters on the observed grains is decreased by 10 and $17 \%$ on average, respectively. The images in figure 2 (d) and (e) compare the region with the near-surface blisters to the unblistered region before the sample is exposed to the deuterium plasma. Most of the grains shown in image (d) are covered with blisters and the grain orientation dependence of relevant blister parameters such as diameter, areal density and coverage is clearly visible. The unblistered region in image (e) shows, except of some dust particles, no particular surface morphology and a well defined grain contrast, similar to a virgin sample surface.

\section{A.2 Blister Characterization}

In order to evaluate the influence of blisters on deuterium transport more detailed information on the blisters is required before the samples are exposed to the deuterium plasma. Especially the depth at which the blisters are located is an important information since it can be directly linked to the deuterium depth profiles measured with NRA. In order to determine the blister depth a FIB cut perpendicular to the sample surface is performed and the obtained cross section is investigated with SEM. Since the relevant blister parameters depend on the grain orientation with respect to the surface, the cut is positioned on several grains exhibiting different blistering behavior. Figure 3 (a) shows an overview of the region where the FIB cut is performed. The cut, indicated by the dash-dotted white line, has a length of approximately $50 \mu \mathrm{m}$ and covers four grains $\mathrm{A}$ to $\mathrm{D}$ with increasing blister size.

Due to the mainly circular shape of the blisters, the diameter is a reasonable quantity for blister comparison. The distribution function of the blister diameter of grain A to D is shown in figure 4 The distribution function of the blister diameter of grain A exhibits a symmetrical shape with a clear maximum. Assuming a normal distribution, the average blister diameter and the corresponding standard derivation are determined to $300 \pm 80 \mathrm{~nm}$. For grain $\mathrm{B}$ the maximum is shifted to a larger diameter and the distribution is slightly asymmetrical with a tail towards small blister diameters. The average blister diameter on grain B is $480 \pm 100 \mathrm{~nm}$. In the cases of grain $C$ and $D$ the distribution functions possess two distinct maxima. In order to account for the presence of a small and a large blister population a combination of two normal distributions is used to fit the data. On grain $C$ the average small and large blister diameters are $350 \pm 150$ and $810 \pm 160 \mathrm{~nm}$, respectively. While on grain D the blisters are slightly larger and the average small and large blister diameters are $380 \pm 140$ and $980 \pm 220 \mathrm{~nm}$, respectively. On both grains, the abundance ratio of small to large blisters is approximately $2: 5$. As the blister size increases from grain A to D, the areal density of the blisters decreases from 3.14 , to 1.98 , to 1.23 , to $0.85 \mu^{-2}$. At the same time the area covered by the blisters increases from 23 , to 35 , to 50 , to $51 \%$.

A magnified section of grain A to D is shown in figure 3 (b) to (e). The bright lines located at the rim of the blisters are caused by an increased emission of secondary electrons and indicate the rupture of the blisters at this positions. This observation agrees well with the FEM simulations by Enomoto et al. [7], which show that the largest stress occurs at the edge of the blisters. In grain A to D the portion of open blisters is $67,57,64$ and $87 \%$, respectively. However, since the cracks, which are only visible as thin lines, are hard to detect and possible subsurface connections between blisters are unknown a quantitative analysis of burst blisters 


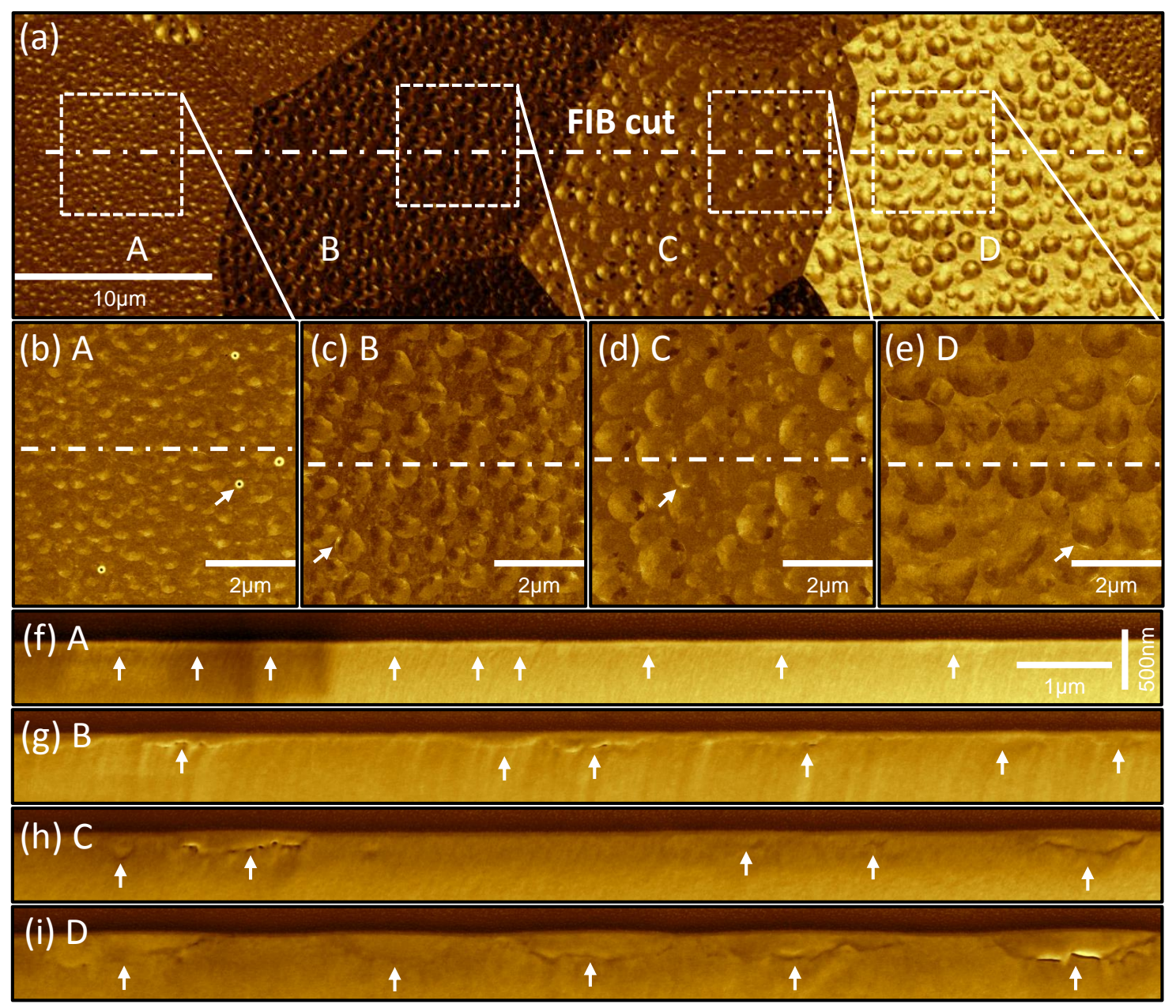

Figure 3: (a) Overview SEM image of four grains A to D with different blister size, sensitive to backscattered electrons. (b) to (e) magnified SEM images of the areas indicated by white dashed boxes, sensitive to secondary electrons. White arrows indicate exemplarily the rupture of blisters at the rim and pores in (b). (f) to (i) SEM images of the cross sections of the different grains A to D along the dash-dotted white lines shown in (b) to (e). The cross section images are obtained under an inclination of $38^{\circ}$, resulting in different horizontal and vertical scales of the images. The white arrows indicate the subsurface cracks of the blisters. 


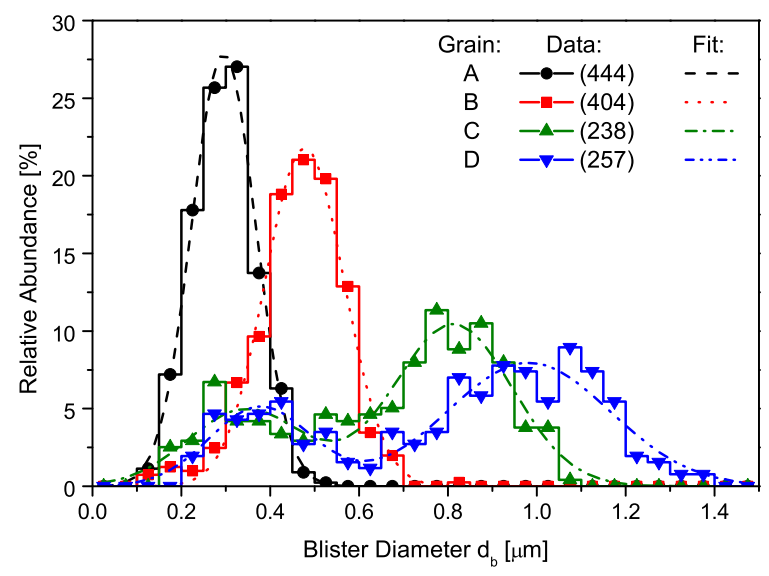

Figure 4: Distribution function of the blister diameter for grains $\mathrm{A}$ to $\mathrm{D}$ in figure 3 (a), with corresponding fits of one respectively two normal distributions. The number of measured blisters is stated in the brackets.

is cumbersome. Hence the stated percentage probably underestimates the amount of blisters with a connection to the surface. Apart from blisters also some pores are observed on grain A, which appear as white circles with black centers in image (b).

The SEM images in figure 3 (f) to (i) show the cross sections prepared by FIB milling of grain A to D along the dash-dotted white lines in the images (b) to (e) in figure 3 under an inclination of $38^{\circ}$. The width of the cracks in the FIB cross section, indicated by white arrows, is not necessarily the diameter of the blisters measured in the topview SEM images, since it is unlikely that all the blisters are cut directly through the centers. Nevertheless, from figure 3 (h) and (i) it can be clearly seen that the cracks which cause the blisters propagate parallel to the surface in a certain depth, only at the blister rim the cracks expand to the surface. Although the blisters are not directly cut in the middle the depth information is still trustworthy. Figure 5 shows the relationship between the blister depth and the blister diameter for grain A to D of the blisters cut by the focused ion beam. Obviously, there exists a general dependence between blister depth and diameter which is independent of the grain orientation with respect to the surface. The larger the depth at which the blisters are initialized, the larger the diameter to which the blisters can grow. A linear fit on the data of all grains shows that the blisters can grow approximately up to a diameter of 5.5 times the depth at which they are created. Looking at the individual grains, it can be seen that the depth distribution at which the blisters occur becomes broader from grain A to D. As a result, the blister size distribution broadens as well, which is in good agreement with the data shown in figure 4 . The average depth at which blisters occur is $57 \pm 6 \mathrm{~nm}$ on grain $A$ and $95 \pm 10 \mathrm{~nm}$ on grain B. In case of grain C and D the two different populations of blisters need to be considered. From figure 4 the transition diameter from small to large blisters can be determined to 500 and $600 \mathrm{~nm}$ on grain $C$ and D, respectively. In figure 5 it can be seen that only two small blisters are cut in grain $C$ and no small blisters are cut in grain D. Hence the average depth of large blisters on grain C and D is found to be $149 \pm 30 \mathrm{~nm}$ and $182 \pm 33 \mathrm{~nm}$. By exploiting the observed general relationship between blister depth and diameter, the average depth of small blisters on grain $C$ and $D$ can be estimated to $63 \pm 27 \mathrm{~nm}$ and $69 \pm 25 \mathrm{~nm}$. The origin of the two blister populations on grain $C$ and $D$ is not yet clear and requires further systematic study. 


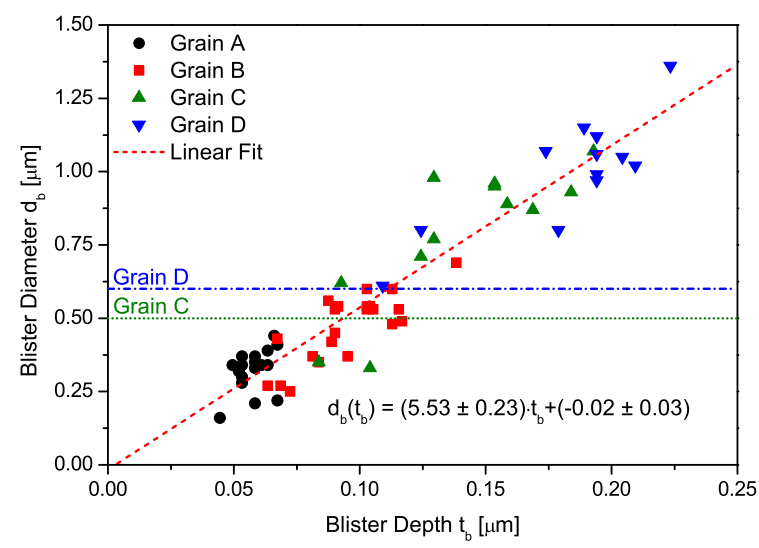

Figure 5: Relationship between blister depth and diameter for the blisters on grain A to D observed in the FIB cut with a linear fit shown as dashed red line. The transition between the small and large blister populations on grain $\mathrm{C}$ and $\mathrm{D}$ are indicated by the dotted green and the dashdotted blue lines.

\section{B. Deuterium Transport}

\section{B.1 Deuterium Depth Profiles}

Figure 6 shows the deuterium depth profiles measured by NRA in the unblistered (a) and blistered (b) region on the sample after exposure to different deuterium fluences. Furthermore, the damage profile calculated by SRIM 2013 for $20 \mathrm{MeV}$ tungsten ions is included as dashed grey line in figure 6. The depth range at which the blisters are located is indicated in figure 6 (b) as grey-shaded area. In case of the unblistered region, shown in figure 6 (a), it can be seen that the deuterium diffusion front propagates within the damaged layer into the bulk as the plasma exposure time is increased. After $2 \mathrm{~h}$ plasma exposure, the deuterium concentration close to the surface, within the first $250 \mathrm{~nm}$, is 0.8 at. $\%$ and decreases to 0.08 at. $\%$ at a depth of $600 \mathrm{~nm}$. For an exposure time of $5 \mathrm{~h}$, also a deuterium concentration of 0.8 at.\% close to the surface is observed. In contrast to the $2 \mathrm{~h}$ exposure the deuterium profile extents to larger depth and a maximum concentration of 1.1 at. $\%$ is reached at approximately $300 \mathrm{~nm}$. As the exposure time is increased the damaged layer becomes subsequently filled with deuterium. After exposing the sample for $40 \mathrm{~h}$ to the plasma, the deuterium depth profile reaches the end of the damaged layer at approximately $2.1 \mu \mathrm{m}$. The $72 \mathrm{~h}$ plasma exposure leads to the decoration of the defects at the end of the damaged layer. Compared to the $40 \mathrm{~h}$ exposure, the deuterium concentration rises in the depth between $1.4 \mu \mathrm{m}$ and $2.1 \mu \mathrm{m}$ from 0.2 at.\% to $0.8 \mathrm{at} . \%$. In addition, deuterium is also found up to $1.3 \mu \mathrm{m}$ behind the damaged layer.

The depth profiles measured in the blistered area of the samples, shown in figure 6 (b), possess a deuterium concentration of 1.1 at.\% within the first $120 \mathrm{~nm}$, which is within the depth where the blisters are located. Furthermore, this near-surface deuterium concentration is reached after $2 \mathrm{~h}$ and appears to be independent of the plasma exposure time. Compared to the depth profiles measured in the unblistered region, the deuterium concentration close to the surface is higher by 0.3 at.\%. For depths larger than $120 \mathrm{~nm}$ the defect concentration in the blistered and unblistered area are expected to be equal due to the same tungsten ion self-damaging procedure. As the plasma exposure time is increased from $2 \mathrm{~h}$ to $72 \mathrm{~h}$, the deuterium in the blistered area diffuses into the bulk and the traps in the damaged layer are subsequently filled. However, compared to the unblistered case, the deuterium front propagates significantly slower into the bulk. An exposure time of $20 \mathrm{~h}$ is required to fill the damaged layer 


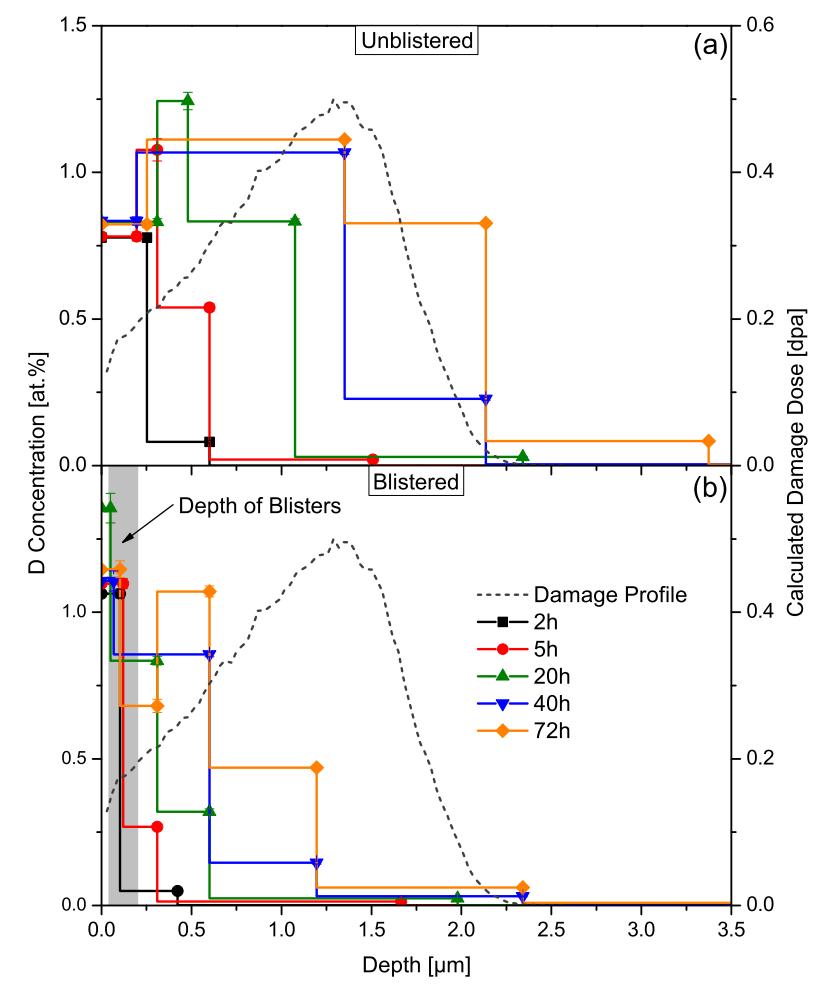

Figure 6: Deuterium depth profiles of (a) unblistered and (b) blistered self-damaged tungsten measured after exposure to different deuterium fluences. The damage profile created by tungsten ions with an energy of $20 \mathrm{MeV}$ (grey dashed line) and the depth range of the blisters (grey-shaded area) are also shown.

behind the $120 \mathrm{~nm}$ blister dominated range up to a concentration of 0.7 at.\% which is found in the unblistered region already after $2 \mathrm{~h}$. Even the longest exposure time of $72 \mathrm{~h}$ is not sufficient for the deuterium front to reach the end of the damaged layer at $2.1 \mu \mathrm{m}$ in the blistered part of the sample. The deuterium depth profile extents approximately $1.2 \mu \mathrm{m}$ into the bulk and the damaged layer is filled to its maximum deuterium concentration of 1.1 at. $\%$ only up to a depth of $0.6 \mu \mathrm{m}$. The comparison of the depth profiles measured in the blistered and unblistered areas show that the presence of blisters close to the surface reduce the deuterium flux into the bulk. Furthermore, this does not occur by enhanced trapping in the blistered zone, but by enhanced re-emission of deuterium. If enhanced trapping would be responsible, then the deuterium which is hindered to diffuse into the bulk must stay within the depth of the blisters. This would result in an considerable increase of the near-surface concentration as the plasma exposure time is prolonged, which is not observed. The observed difference of 0.3 at. $\%$ in the near-surface deuterium concentration is most likely due to a higher concentration of defects in the proximity of the blisters, which have not entirely recovered in the sample annealing step.

\section{B.2 Deuterium Retention}

The deuterium retention, derived by integration of the depth profiles, is shown in figure 7 as a function of the plasma exposure time for the blistered and unblistered areas of the samples. In addition, the ratio of the deuterium retention in the blistered and unblistered regions is also plotted in figure 7. As already expected from the depth profiles in figure 6, the deuterium retention in the blistered area is significantly lower. After a plasma exposure time of $72 \mathrm{~h}$, 


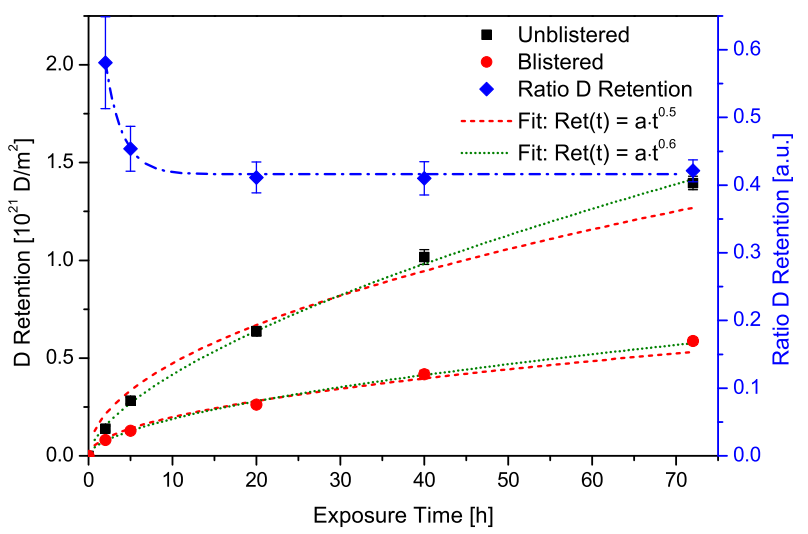

Figure 7: Deuterium retention in blistered and unblistered areas shown as solid red circles and solid black squares with the corresponding power law fits indicated as dotted green lines and dashed red lines. Ratio of deuterium retention in blistered to unblistered areas plotted as solid blue diamonds with dash-dotted blue line to guide the eye.

the deuterium retention is $0.6 \cdot 10^{21} \mathrm{D} / \mathrm{m}^{2}$ and $1.4 \cdot 10^{21} \mathrm{D} / \mathrm{m}^{2}$ in the blistered and unblistered regions, respectively. This difference implies a considerably enhanced re-emission of deuterium in the blistered area. Despite the difference in the absolute deuterium retention, the dependence of the retention on the exposure time is very similar in both cases. However, the ratio of deuterium retention in the blistered to the unblistered area, shown in figure 77, is not constant but is 0.58 for the shortest exposure time and decreases to 0.41 as the exposure is prolonged. This observation could be explained by open blisters located at a finite depth and a highly trap-dominated diffusion of deuterium in the damaged layer as follows: For very short exposure times, at which the deuterium diffusion front has not yet propagated to the depth of the blisters, the deuterium retention in the blistered and the unblistered area should be equal and an ratio of one is expected. The underlying reason is simply no deuterium atom has encountered an open blister yet and therefore the deuterium flux into the bulk is not reduced. As soon as the deuterium diffusion front reaches the depth region of the blisters, deuterium captured by open blisters is re-emitted from the sample. This effect reduces the amount of deuterium which passes the blistered zone and diffuses into the bulk. As less deuterium enters the bulk compared to the unblistered case, a lower retention is the consequence and the retention ratio, shown in figure 7, decreases. Taking the grain orientation dependent blister depth and the fact that NRA averages over many grains into account, a certain depth range of blisters, indicated by the grey-shaded area in figure 6 (b), is obtained. As long as the deuterium diffusion front propagates within this range, the mean flux into the bulk and the retention declines with respect to the unblistered case. This effect is clearly seen in the $2 \mathrm{~h}$ and $5 \mathrm{~h}$ plasma exposure, where the deuterium retention ratio reduces from 0.58 to 0.45 . Once the diffusion front has passed the depth range of the blisters, the deuterium fluxes into the bulk in the blistered and unblistered area do not change anymore with respect to each other. Hence the ratio of deuterium retention in the blistered to the unblistered area converges against a certain value, which is 0.41 in this case. Because the damaged layer has a finite thickness and therefore a finite total capacity, the ratio of deuterium retention is expected to increase again, once the damaged layer in the unblistered area is saturated with deuterium. As the damaged layer in the blistered area becomes increasingly filled with deuterium, the retention ratio is expected to converge to almost unity. 
As previously described in the introduction, the proposed mechanisms how near-surface blisters can influence the deuterium transport into the bulk can be divided into two categories: Enhanced trapping and enhanced re-emission. Taking the results obtained from the SEM investigation and the NRA measurements into account, it can be concluded that the here observed reduction of the deuterium flux into the bulk is the result of an increased re-emission of deuterium from the sample caused by ruptured blisters which provide shortcuts to the surface.

\section{B.3 Analytical Hydrogen Retention Model}

In order gain a quantitative understanding of the blister induced re-emission flux and the reduction of the flux into the bulk, a simple analytical model to describe hydrogen retention in tungsten based on Schmid [35] is applied. A schematic picture of the model and the relevant assumptions are shown in figure 8 . Hydrogen with a flux of $\Gamma_{i m p}$ is implanted into a semi-

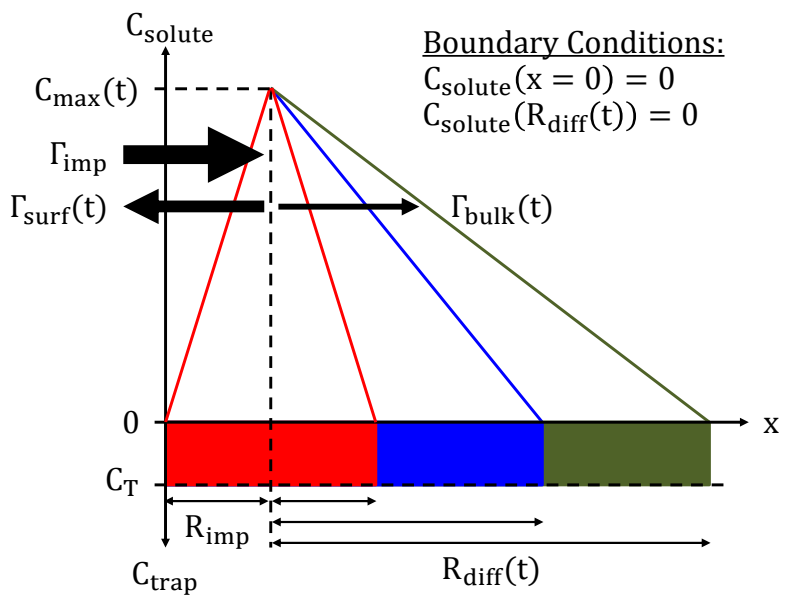

Figure 8: Schematic picture of the analytical model to describe hydrogen retention in tungsten based on Schmid [35.

infinite sample at a depth $R_{i m p}$ from the surface assuming a delta function as implantation profile. The trap concentration profile is assumed to be constant and filled to an equilibrium level $C_{T}$ at a respective exposure temperature. Furthermore, the model assumes a diffusion limited boundary condition at the surface and a moving absorbing boundary condition at $R_{\text {diff }}(t)$. The latter accounts for a strongly trap-dominated diffusion, which requires the traps to be filled before the solute front can propagate further into the bulk. As a consequence the retention Ret $(t)$ can be expressed as,

$$
\operatorname{Ret}(t)=C_{T} \cdot \rho\left(R_{\text {imp }}+R_{\text {diff }}(t)\right),
$$

with $\rho$ corresponding to the number density of tungsten. In the initial phase of implantation the hydrogen concentration $C_{\max }(t)$ at $R_{i m p}$ builds up and thereby generates a flux of hydrogen into the bulk $\Gamma_{\text {bulk }}(t)$ and to the surface $\Gamma_{\text {surf }}(t)$. Both fluxes are equal at the beginning until the solute front reaches the surface, which pins the solute concentration to zero due to the diffusion limited boundary condition. At this point the solute front has propagated another $R_{\text {imp }}$ deep into the bulk, as illustrated by the red equal-sided triangle in figure 8 This situation represents the initial state from which the temporal evolution of the system is studied. In this case the flux balance of the model can be written as,

$$
\Gamma_{\text {imp }}=\Gamma_{\text {surf }}(t)+\Gamma_{\text {bulk }}(t),
$$


with the fluxes to the surface $\Gamma_{\text {surf }}(t)$ and into the bulk $\Gamma_{\text {bulk }}(t)$ as a function of the solute gradient from $R_{\text {imp }}$ to the surface and to $R_{\text {diff }}(t)$,

$$
\begin{aligned}
& \Gamma_{\text {surf }}(t)=\frac{D(T) \cdot \rho \cdot C_{\max }(t)}{R_{\text {imp }}}, \\
& \Gamma_{\text {bulk }}(t)=\frac{D(T) \cdot \rho \cdot C_{\max }(t)}{R_{\text {diff }}(t)},
\end{aligned}
$$

with $D(T)$ being the diffusion coefficient. The maximum solute concentration $C_{\max }(t)$ can be obtained by inserting equations (3) and (4) into equation (2) which yields:

$$
C_{\text {max }}(t)=\frac{\Gamma_{\text {imp }}}{D(T) \cdot \rho} \cdot \frac{R_{\text {imp }} \cdot R_{\text {diff }}(t)}{R_{\text {diff }}(t)+R_{\text {imp }}} .
$$

Furthermore, the integrated flux into the bulk needs to correspond to the bulk retention, which provides the following condition:

$$
\rho \cdot C_{T} \cdot R_{\text {diff }}(t)=\int \Gamma_{\text {bulk }}(t) d t .
$$

Inserting equation (5) in the flux balance equation (4), allows to derive an expression of $\Gamma_{b u l k}(t)$ only depending on diffusion length $R_{\text {diff }}(t)$, which can be used in equation (6) to obtain the following differential equation for $R_{\text {diff }}(t)$ :

$$
\frac{d R_{\text {diff }}(t)}{d t}=\frac{\Gamma_{i m p} \cdot R_{i m p}}{\rho \cdot C_{T}} \cdot \frac{1}{R_{\text {imp }}+R_{\text {diff }}(t)}
$$

Solving equation $(7)$ with the boundary condition $R_{\text {diff }}(0)=R_{\text {imp }}$ results in the following expressions for $R_{\text {diff }}(t)$,

$$
R_{\text {diff }}(t)=R_{\text {imp }}\left(-1+\frac{1}{\beta} \sqrt{4 \beta^{2}+2 \beta \Gamma_{i m p} \cdot t}\right),
$$

with $\beta=R_{i m p} \cdot C_{T} \cdot \rho$, which is essentially the hydrogen retention between the surface and $R_{i m p}$. Substituting $R_{\text {diff }}(t)$ in equation (1) with expression (8) allows to write the retention $\operatorname{Ret}(t)$ as:

$$
\operatorname{Ret}(t)=\sqrt{4 \beta^{2}+2 \beta \cdot \Gamma_{i m p} \cdot t} .
$$

Furthermore, by replacing $R_{\text {diff }}(t)$ in equation (5) with equation (8), $C_{\max }(t)$ can be expressed by:

$$
C_{\text {max }}(t)=\frac{\Gamma_{i m p} \cdot R_{i m p}}{D(T) \cdot \rho} \cdot\left(1-\frac{\beta}{\sqrt{4 \beta^{2}+2 \beta \Gamma_{i m p} \cdot t}}\right) .
$$

The solution of $C_{\max }(t)$ and $R_{\text {diff }}(t)$ can be used in equation (3) and (4) to determine $\Gamma_{\text {bulk }}(t)$ and $\Gamma_{\text {surf }}(t)$ to

$$
\Gamma_{\text {bulk }}(t)=\Gamma_{\text {imp }} \cdot \frac{\beta}{\sqrt{4 \beta^{2}+2 \beta \Gamma_{\text {imp }} \cdot t}}=\Gamma_{\text {imp }} \cdot \frac{\beta}{\operatorname{Ret}(t)}
$$

and

$$
\Gamma_{\text {surf }}(t)=\Gamma_{\text {imp }} \cdot\left(1-\frac{\beta}{\sqrt{4 \beta^{2}+2 \beta \Gamma_{i m p} \cdot t}}\right)=\Gamma_{i m p} \cdot\left(1-\frac{\beta}{\operatorname{Ret}(t)}\right) .
$$

Considering the time evolution of the system shown in figure 8 with the obtained equations (8) to (12), it can be seen that the diffusion length $R_{\text {diff }}(t)$ increases in a square-root-like 
fashion, while $C_{\max }(t)$ saturates as the exposure time tends to infinity. As a result, the solute concentration gradient from $R_{\text {imp }}$ into the bulk converges to zero and therefore also the flux $\Gamma_{\text {bulk }}(t) . C_{\max }(t)$ increases until the solute gradient between $R_{i m p}$ and the surface is large enough that $\Gamma_{\text {surf }}(t)$ compensates $\Gamma_{\text {imp }}$. Since the hydrogen retention depends linearly on the diffusion length, $\operatorname{Ret}(t)$ increases in a square-root-like fashion as a function of time $t$ and implanted flux $\Gamma_{i m p}$.

In order to describe the presence of blisters, which drain hydrogen from the sample, an additional loss channel is introduced by reducing $\Gamma_{i m p}$ by $\Gamma_{\text {sink }}$. This Ansatz is of course a simplification, since it implies that the blisters are located at the implantation depth $R_{i m p}$ which is not the case. Nevertheless, the hydrogen retention $\operatorname{Ret}_{u b}(t)$ and $\operatorname{Ret}_{b}(t)$ for the unblistered and blistered case can be expressed by:

$$
\begin{gathered}
\operatorname{Ret}_{u b}(t)=\sqrt{4 \beta^{2}+2 \beta \cdot \Gamma_{i m p} \cdot t} \approx \sqrt{2 \beta \cdot \Gamma_{i m p} \cdot t} \\
\operatorname{Ret}_{b}(t)=\sqrt{4 \beta^{2}+2 \beta \cdot\left(\Gamma_{i m p}-\Gamma_{\text {sink }}\right) \cdot t} \approx \sqrt{2 \beta \cdot\left(\Gamma_{i m p}-\Gamma_{\text {sink }}\right) \cdot t} .
\end{gathered}
$$

Taking the experimental conditions into account, $4 \beta^{2}$ is of the same order of magnitude as $2 \beta \Gamma_{i m p}$. Hence the retention becomes quickly dominated by the time dependent term, which justifies the approximation in equations (13) and (14). Dividing equation (14) by (13) the hydrogen flux re-emitted by the blisters can be determined to

$$
\Gamma_{\text {sink }}=\Gamma_{i m p} \cdot\left(1-\left(\frac{\operatorname{Ret}_{b}}{\operatorname{Ret}_{u b}}\right)^{2}\right) .
$$

In the case of blisters the flux into the bulk in equation (11) becomes:

$$
\Gamma_{\text {bulkb }}(t)=\left(\Gamma_{\text {imp }}-\Gamma_{\text {sink }}\right) \cdot \frac{\beta}{\operatorname{Ret}_{b}(t)} .
$$

Together with equations (15) and (11) the relation between the hydrogen flux into the bulk in blistered and unblistered area can be expressed as:

$$
\Gamma_{b u l k b}(t)=\frac{\operatorname{Ret}_{b}(t)}{\operatorname{Ret}_{u b}(t)} \cdot \Gamma_{b u l k u b}(t) .
$$

Fitting the experimental deuterium retention data with a square-root function, plotted as dashed red lines in figure 7 and applying equation (15) allows to determine the ratio between $\Gamma_{\text {sink }}$ to $\Gamma_{i m p}$ to $0.82 \pm 0.03$. However, the square-root fit does not provide the best approximation of the data. The deuterium retention is overestimated at shorter and underestimated at longer exposure times. Treating the exponent also as free fit parameter an exponent of $0.62 \pm 0.02$ and $0.57 \pm 0.02$ is obtained for the unblistered and blistered case. Fitting the two data sets with a mean exponent of $0.60 \pm 0.03$, illustrated as green doted line in figure 7 and using equation (15) with an adapted exponent, results in a ratio of $0.76 \pm 0.02$ between $\Gamma_{\text {sink }}$ and $\Gamma_{i m p}$. Hence, within the framework of the model, the additional loss flux $\Gamma_{\text {sink }}$ amounts to approximately $80 \%$ of the implanted flux $\Gamma_{i m p}$. Furthermore, by extrapolating the fit, with the mean exponent of $0.60 \pm 0.03$, the required exposure time to fill the damaged layer in the blistered area can be determined to about $320 \mathrm{~h}$.

From equation (17) it can be seen that the ratio of the deuterium flux into the bulk in the blistered to the unblistered area equals the ratio of the corresponding deuterium retentions shown in figure 7 At the beginning of the deuterium implantation, e.g. after $2 \mathrm{~h}$, and $5 \mathrm{~h}$, when not all open blisters participate in the re-emission of deuterium, the flux into the bulk in the blistered region is 0.58 , respectively 0.45 times the flux into the bulk in the unblistered region. When all open blisters contribute to the deuterium re-emission this ratio becomes 0.41 . Hence it can be concluded, that the presence of open blisters lead to a reduction of the deuterium bulk flux by almost $60 \%$. 


\section{Conclusion}

The influence of near-surface blisters on the transport of deuterium in tungsten is studied by combining scanning electron microscopy with nuclear reaction analysis. A blistered region is first created on the samples by $\mathrm{keV}$ ion implantation of hydrogen isotopes at $150 \mathrm{~K}$. Annealing of the samples to $923 \mathrm{~K}$ results in the release of the hydrogen isotopes while preserving the blister structure. Before the partly blistered samples are exposed to a deuterium plasma under gentle loading conditions, a defect-rich layer, which serves as a getter layer for deuterium, is created by $20 \mathrm{MeV}$ tungsten ion irradiation. Characterization of the blisters by SEM assisted by FIB revealed grain orientation dependent formation of circular-shaped blisters, with diameters up to $1.2 \mu \mathrm{m}$ at depths up to $220 \mathrm{~nm}$. Furthermore, the majority of the observed blisters are ruptured at the blister rim. NRA depth profiling in the blistered and unblistered regions of the samples revealed that the diffusion of deuterium into the bulk is significantly reduced by the presence of blisters close to the surface. The re-emission of deuterium from the sample by ruptured blisters is identified as the dominant effect. Based on a simple analytical model, approximately $80 \%$ of the implanted deuterium flux is released by open blisters and the deuterium flux into the bulk is reduced by almost $60 \%$. It has been demonstrated in this article that the surface morphology can have a strong influence on the deuterium uptake and the retention in tungsten if saturation is not yet reached.

It is important to note that the final retention, after the sample is completely saturated with deuterium, is not directly influenced by the presence of open blisters. Simply because the retention is ultimately determined by the available total number of traps which can be occupied at a given temperature. The only direct effect of open blisters is the prolongation of the required time to reach saturation of the deuterium retention. Indirectly, the presence of open blisters increases the retention due to the enhanced defect concentration in the proximity of the blisters. However, as the here performed experiment shows, open blisters reduce the deuterium flux into the bulk. When the diffusion front reaches the rear side of the sample the bulk flux becomes the permeation flux. Thus the existence of open blisters at the front side of the sample reduces the permeation flux. In order to evaluate the relationship between the deuterium flux into the bulk and the blister parameters, such as diameter, areal density, surface coverage and depth, the experiment cannot be performed on polycrystalline material due to the grain orientation dependence of those parameters. Hence a systematic study on single crystals would be reasonable.

It should be kept in mind, that in the here conducted experiment the surface morphology is essentially decoupled from the deuterium retention study. Before the deuterium is inserted into the samples the surface morphology, consisting of densely packed predominately open blisters, has already been established. In addition most of the defects in the proximity of the blisters have been recovered by annealing the samples. The retaining effect of the blisters is therefore reduced and the effect of open blisters appears clearer. Since the deuterium plasma loading is conducted under very gentle conditions no additional defect creation is expected. Hence during the plasma exposure an existing trap concentration profile which is assumed to be time independent is filled with deuterium.

The situation is different when the deuterium retention is studied by plasma loading, respectively ion beam implantation under conditions which alter the surface morphology of the sample. Under this circumstance the formation, growth and rupture of blisters as well as the defects associated with blisters evolve in parallel to the deuterium retention. This is often the case for deuterium retention studies performed in linear plasma devices, where surface modifications, such as cracks, nanostructures and blisters are frequently observed after sample exposure [14, 36]. It needs to be evaluated to which extent the here observed effect of open blisters on deuterium uptake affects retention studies carried out in linear plasma devices.

For a future nuclear fusion device the situation is even more complex. In addition to hydrogen isotopes also other ion species, e.g. helium, nitrogen and argon, impinge on the 
technical tungsten surface. It has been shown by Nishijima et al. [13] that technical tungsten surfaces are less prone to blistering than polished ones. However, new results by Manhard et al. [37] reveal that the blister formation on technical surfaces is not entirely suppressed. Furthermore, experiments exploring synergistic effects of deuterium and helium [13, 38, 39] found a reduction of blistering by helium pre- and co-implantation with deuterium. The question whether blistering is an issue for future fusion devices is not entirely solved, but their appearance might not only be a disadvantage with respect to the tritium inventory. Retention in the depth of the blisters would be enhanced, but at the same time permeation of tritium would be suppressed. Moreover, the here observed physical principle, namely the reduction of the deuterium flux into the bulk by enhanced re-emission through shortcuts to the surface, is not exclusively bound to open blisters. An intentional surface modification e.g. by trenches, drain pipes, or castellation, which have a similar effect, could be applied to reduce the permeation flux of tritium to the coolant.

\section{ACKNOWLEDGMent}

We want to thank G. Matern for operating the SEM and FIB system, which enabled us to investigate the surface morphology and T. Dürbeck for his assistance with the annealing of the samples. Furthermore, we are grateful for the technical support and the operation of the tandem accelerator at IPP Garching by M. Fußeder and J. Dorner, which allowed us to perform the tungsten ion self-damaging and the NRA measurements. Finally, we appreciate the fruitful scientific discussions with W. Jacob. This work has been carried out within the framework of the EUROfusion Consortium and has received funding from the Euratom research and training programme $2014-2018$ under grant agreement No 633053. The views and opinions expressed herein do not necessarily reflect those of the European Commission. The work was partially carried out under WP PFC.

\section{REFERENCES}

[1] J. Roth, E. Tsitrone, A. Loarte, T. Loarer, G. Counsell, R. Neu, V. Philipps, S. Brezinsek, M. Lehnen, P. Coad, C. Grisolia, K. Schmid, K. Krieger, A. Kallenbach, B. Lipschultz, R. Doerner, R. Causey, V. Alimov, W. Shu, O. Ogorodnikova, A. Kirschner, G. Federici, and A. Kukushkin, "Recent analysis of key plasma wall interactions issues for ITER," Journal of Nuclear Materials, vol. 390-391, no. 0, pp. 1-9, Jun. 2009. [Online]. Available: http://www.sciencedirect.com/science/article/pii/S0022311509000506

[2] V. Barabash, G. Federici, J. Linke, and C. Wu, "Material/plasma surface interaction issues following neutron damage," Journal of Nuclear Materials, vol. 313-316, pp. 42 - 51, 2003, plasma-Surface Interactions in Controlled Fusion Devices 15. [Online]. Available: http://www.sciencedirect.com/science/article/pii/S0022311502013302

[3] H. Bolt, V. Barabash, G. Federici, J. Linke, A. Loarte, J. Roth, and K. Sato, "Plasma facing and high heat flux materials - needs for ITER and beyond," Journal of Nuclear Materials, vol. 307-311, Part 1, pp. 43 - 52, 2002. [Online]. Available: http://www.sciencedirect.com/science/article/pii/S0022311502011753

[4] K. Tokunaga, M. Baldwin, R. Doerner, N. Noda, Y. Kubota, N. Yoshida, T. Sogabe, T. Kato, and B. Schedler, "Blister formation and deuterium retention on tungsten exposed to low energy and high flux deuterium plasma," Journal of Nuclear Materials, vol. 337 - 339, pp. 887 - 891, 2005, pSI-16. [Online]. Available: //www.sciencedirect.com/science/article/pii/S0022311504009298 
[5] G.-N. Luo, W. Shu, and M. Nishi, "Influence of blistering on deuterium retention in tungsten irradiated by high flux deuterium 10-100 ev plasmas," Fusion Engineering and Design, vol. 81, pp. 957 - 962, 2006. [Online]. Available: //www.sciencedirect.com/science/article/pii/S0920379605006125

[6] V. Alimov, W. Shu, J. Roth, S. Lindig, M. Balden, K. Isobe, and T. Yamanishi, "Temperature dependence of surface topography and deuterium retention in tungsten exposed to low-energy, high-flux d plasma," Journal of Nuclear Materials, vol. 417, no. 1-3, pp. 572 - 575, 2011, proceedings of ICFRM-14. [Online]. Available: http://www.sciencedirect.com/science/article/pii/S0022311511001206

[7] N. Enomoto, S. Muto, T. Tanabe, J. Davis, and A. Haasz, "Grazing-incidence electron microscopy of surface blisters in single- and polycrystalline tungsten formed by $\mathrm{H}^{+}, \mathrm{D}^{+}$and $\mathrm{He}^{+}$irradiation," Journal of Nuclear Materials, vol. 385, no. 3, pp. 606 - 614, 2009. [Online]. Available: http://www.sciencedirect.com/science/article/pii/ S0022311509003213

[8] G.-N. Luo, W. Shu, and M. Nishi, "Incident energy dependence of blistering at tungsten irradiated by low energy high flux deuterium plasma beams," Journal of Nuclear Materials, vol. 347, no. 1-2, pp. 111 - 117, 2005. [Online]. Available: http://www.sciencedirect.com/science/article/pii/S0022311505003752

[9] M. 't Hoen, M. Balden, A. Manhard, M. Mayer, S. Elgeti, A. Kleyn, and P. Z. van Emmichoven, "Surface morphology and deuterium retention of tungsten after low- and high-flux deuterium plasma exposure," Nuclear Fusion, vol. 54, no. 8, p. 083014, 2014. [Online]. Available: http://stacks.iop.org/0029-5515/54/i=8/a=083014

[10] L. Buzi, G. D. Temmerman, B. Unterberg, M. Reinhart, A. Litnovsky, V. Philipps, G. V. Oost, and S. Möller, "Influence of particle flux density and temperature on surface modifications of tungsten and deuterium retention," Journal of Nuclear Materials, vol. 455, no. 1-3, pp. 316 - 319, 2014, proceedings of the 16th International Conference on Fusion Reactor Materials (ICFRM-16). [Online]. Available: http://www.sciencedirect.com/science/article/pii/S0022311514004164

[11] H. Xu, Y. Zhang, Y. Yuan, B. Fu, A. Godfrey, G. D. Temmerman, W. Liu, and X. Huang, "Observations of orientation dependence of surface morphology in tungsten implanted by low energy and high flux D plasma," Journal of Nuclear Materials, vol. 443, no. 1-3, pp. 452 - 457, 2013. [Online]. Available: http://www.sciencedirect.com/science/article/pii/S0022311513009501

[12] G.-N. Luo, K. Umstadter, W. Shu, W. Wampler, and G.-H. Lu, "Behavior of tungsten with exposure to deuterium plasmas," Nuclear Instruments and Methods in Physics Research Section B: Beam Interactions with Materials and Atoms, vol. 267, no. 18, pp. 3041 - 3045, 2009, proceedings of the Ninth International Conference on Computer Simulation of Radiation Effects in Solids. [Online]. Available: http://www.sciencedirect.com/science/article/pii/S0168583X09007150

[13] D. Nishijima, H. Iwakiri, K. Amano, M. Ye, N. Ohno, K. Tokunaga, N. Yoshida, and S. Takamura, "Suppression of blister formation and deuterium retention on tungsten surface due to mechanical polishing and helium pre-exposure," Nuclear Fusion, vol. 45, no. 7, p. 669, 2005. [Online]. Available: http://stacks.iop.org/0029-5515/45/i=7/a=016

[14] W. Shu, E. Wakai, and T. Yamanishi, "Blister bursting and deuterium bursting release from tungsten exposed to high fluences of high flux and low energy deuterium plasma," Nuclear Fusion, vol. 47, no. 3, pp. 201-, 2007. [Online]. Available: http://stacks.iop.org/0029-5515/47/i=3/a=006 
[15] Y. Jia, G. D. Temmerman, G.-N. Luo, H. Xu, C. Li, B. Fu, and W. Liu, "Surface morphology and deuterium retention in tungsten exposed to high flux D plasma at high temperatures," Journal of Nuclear Materials, vol. 457, pp. 213 - 219, 2015. [Online]. Available: http://www.sciencedirect.com/science/article/pii/S0022311514008824

[16] S. Lindig, M. Balden, V. K. Alimov, T. Yamanishi, W. M. Shu, and J. Roth, "Subsurface morphology changes due to deuterium bombardment of tungsten," Physica Scripta, vol. 2009, no. T138, pp. 014040-, 2009. [Online]. Available: http://stacks.iop.org/1402-4896/2009/i=T138/a=014040

[17] J. Condon and T. Schober, "Hydrogen bubbles in metals," Journal of Nuclear Materials, vol. 207, pp. 1 - 24, 1993. [Online]. Available: http://www.sciencedirect.com/science/article/ pii/002231159390244S

[18] A. Haasz, M. Poon, and J. Davis, "The effect of ion damage on deuterium trapping in tungsten," Journal of Nuclear Materials, vol. 266 - 269, pp. 520 - 525, 1999. [Online]. Available: // www.sciencedirect.com/science/article/pii/S0022311598005868

[19] T. Venhaus, R. Causey, R. Doerner, and T. Abeln, "Behavior of tungsten exposed to high fluences of low energy hydrogen isotopes," Journal of Nuclear Materials, vol. 290 - 293, pp. 505 - 508, 2001, 14th Int. Conf. on Plasma-Surface Interactions in Controlled Fusion D evices. [Online]. Available:///www.sciencedirect.com/science/article/pii/S0022311500004438

[20] M. Zibrov, M. Balden, T. Morgan, and M. Mayer, "Deuterium trapping and surface modification of polycrystalline tungsten exposed to a high-flux plasma at high fluences," Nuclear Fusion, Accepted.

[21] L. Gao, U. von Toussaint, W. Jacob, M. Balden, and A. Manhard, "Suppression of hydrogen-induced blistering of tungsten by pre-irradiation at low temperature," Nuclear Fusion, vol. 54, no. 12, p. 122003, 2014. [Online]. Available: http: //stacks.iop.org/0029-5515/54/i=12/a=122003

[22] A. Manhard, U. von Toussaint, M. Balden, S. Elgeti, T. Schwarz-Selinger, L. Gao, S. Kapser, T. Płociński, J. Grzonka, M. Gloc, and L. Ciupiński, "Microstructure and defect analysis in the vicinity of blisters in polycrystalline tungsten," Nuclear Materials and Energy, pp. -, 2016. [Online]. Available: http: //www.sciencedirect.com/science/article/pii/S2352179116300369

[23] I. Bizyukov and K. Krieger, "Dual beam experiment for simultaneous irradiation of surfaces with ion species of gaseous and solid-state elements," Review of Scientific Instruments, vol. 77, no. 4, pp. 043501-043501-10, 2006.

[24] E. Salançon and T. Dürbeck and T. Schwarz-Selinger and F. Genoese and W. Jacob, "Redeposition of amorphous hydrogenated carbon films during thermal decomposition," Journal of Nuclear Materials, vol. 376, no. 2, pp. 160 - 168, 2008. [Online]. Available: //www.sciencedirect.com/science/article/pii/S0022311508001633

[25] T. Schwarz-Selinger, "Deuterium retention in mev self-implanted tungsten: influence of damaging dose rate," Nuclear Materials and Energy, Accepted.

[26] Standard Practice for Neutron Radiation Damage Simulation by Charged-Particle Irradiation, ASTM International Std.

[27] B. Tyburska, V. Alimov, O. Ogorodnikova, K. Schmid, and K. Ertl, "Deuterium retention in self-damaged tungsten," Journal of Nuclear Materials, vol. 395, no. 1-3, pp. 150 - 155, 2009. [Online]. Available: http://www.sciencedirect.com/science/article/pii/ S002231150900854X 
[28] A. Manhard, T. Schwarz-Selinger, and W. Jacob, "Quantification of the deuterium ion fluxes from a plasma source," Plasma Sources Science and Technology, vol. 20, no. 1, p. 015010, 2011. [Online]. Available: http://stacks.iop.org/0963-0252/20/i=1/a=015010

[29] V. Alimov, M. Mayer, and J. Roth, "Differential cross-section of the $\mathrm{D}\left({ }^{3} \mathrm{He}, \mathrm{p}\right)^{4} \mathrm{He}$ nuclear reaction and depth profiling of deuterium up to large depths," Nuclear Instruments and Methods in Physics Research Section B: Beam Interactions with Materials and Atoms, vol. 234, no. 3, pp. 169-175, Jun. 2005. [Online]. Available: http://www.sciencedirect.com/science/article/pii/S0168583X05000121

[30] B. Wielunska, M. Mayer, and T. Schwarz-Selinger, "Optimization of the depth resolution for deuterium depth profiling up to large depths," Nuclear Instruments and Methods in Physics Research Section B: Beam Interactions with Materials and Atoms, vol. 387, pp. 103 - 114, 2016. [Online]. Available: http://www.sciencedirect.com/science/article/pii/ S0168583X16303780

[31] M. Mayer, E. Gauthier, K. Sugiyama, and U. von Toussaint, "Quantitative depth profiling of deuterium up to very large depths," Nuclear Instruments and Methods in Physics Research Section B: Beam Interactions with Materials and Atoms, vol. 267, no. 3, pp. 506 - 512, 2009. [Online]. Available: http: //www.sciencedirect.com/science/article/pii/S0168583X08013050

[32] M. Mayer, "SIMNRA User's Guide," Max-Planck-Institut für Plasmaphysik IPP 9/113, 1997.

[33] K. Schmid and U. von Toussaint, "Statistically sound evaluation of trace element depth profiles by ion beam analysis," Nuclear Instruments and Methods in Physics Research Section B: Beam Interactions with Materials and Atoms, vol. 281, no. 0, pp. 64 - 71, 2012. [Online]. Available: http://www.sciencedirect.com/science/article/pii/S0168583X12002042

[34] F. Maury, M. Biget, P. Vajda, A. Lucasson, and P. Lucasson, "Frenkel pair creation and stage I recovery in W crystals irradiated near threshold," Radiation Effects, vol. 38, no. 1-2, pp. 53-65, 1978. [Online]. Available: http://dx.doi.org/10.1080/00337577808233209

[35] K. Schmid, "Diffusion-trapping modelling of hydrogen recycling in tungsten under ELM-like heat loads," Physica Scripta, vol. 2016, no. T167, p. 014025, 2016. [Online]. Available: http://stacks.iop.org/1402-4896/2016/i=T167/a=014025

[36] H. Xu, G. Luo, H. Schut, Y. Yuan, B. Fu, A. Godfrey, W. Liu, and G. Temmerman, "Enhanced modification of tungsten surface by nanostructure formation during high flux deuterium plasma exposure," Journal of Nuclear Materials, vol. 447, no. 1-3, pp. 22-27, Apr. 2014. [Online]. Available: http: //www.sciencedirect.com/science/article/pii/S0022311513012968

[37] A. Manhard, M. Balden, U. von Toussaint, J. Bauer, L. Gao, and K. Schmid, "Blistering and deuterium uptake of tungsten with rough and technical surfaces," in $13^{\text {th }}$ International Conference on Diffusion in Solids and Liquids, 2017.

[38] M. Miyamoto, D. Nishijima, Y. Ueda, R. Doerner, H. Kurishita, M. Baldwin, S. Morito, K. Ono, and J. Hanna, "Observations of suppressed retention and blistering for tungsten exposed to deuterium-helium mixture plasmas," Nuclear Fusion, vol. 49, no. 6, p. 065035, 2009. [Online]. Available: http://stacks.iop.org/0029-5515/49/i=6/a=065035

[39] V. K. Alimov, W. M. Shu, J. Roth, K. Sugiyama, S. Lindig, M. Balden, K. Isobe, and T. Yamanishi, "Surface morphology and deuterium retention in tungsten exposed to low-energy, high flux pure and helium-seeded deuterium plasmas," 
Physica Scripta, vol. 2009, no. T138, pp. 014048-, 2009. [Online]. Available: http:/ /stacks.iop.org/1402-4896/2009/i=T138/a=014048 